\title{
Satellite glial cells of the peripheral nervous system
}

\section{Introduction}

Dorsal root ganglia (DRG) and Trigeminal ganglia (TG) neurons are the primary afferents transmitting sensory information from the periphery to the central nervous system. DRGs are situated on posterior roots of spinal nerves, TGs are on the cerebral surface of the temporal bone. Neurons in these ganglia have unique pseudo-unipolar structure (Figure 1). The cell body (soma) of each neurongives out one axon that bifurcates into two branches. The peripheral branch travels to skin, viscera and orofacial areas. The central branch travels to the dorsal horn of the spinal cord or the medullary dorsal horns of the trigeminal spinal tract nucleus. Afferent spikes to somata provides information for protein synthesis and maintenance of optimal levels of receptors and ion channels in both nerve terminals. Activity in somata dynamically contributes to nerve signaling to dorsal horn cells. Nonneuronal cells also affect neuronal activity through glial-neuron
Volume 5 Issue 2 - 202I

\author{
David John Mackay Smith \\ University of Queensland,Australia
}

Correspondence: David John Mackay Smith, University of

Queensland,Australia, Email djmsmith8@bigpond.com

Received: February 24, 2021 | Published: May 12, 2021

interaction. Somata of sensory neurons do not form synaptic contacts with one another in the ganglia, instead they are enveloped by satellite glial cells (SGCs).
Figure I DRG and DRG neuron.

Satellite glial cells envelop the somata of peripheral sensory and autonomicnerves. Located in sensory, sympathetic and parasympathetic ganglia where they form a thin, tight sheath around each individual neural soma. A narrow gap, the approximate width of a synaptic cleft $(20 \mathrm{~nm})$ between the neuronal stoma and the SGC enables control of the extracellular space and allows rapid bidirectional communication. SGCs are homeostatic cells supporting and modulating the activity of sensory neurons by expression of glutamate transporters and enzymes involved in the glutamate-glutamine cycle and buffering potassium to maintain an appropriate $\mathrm{K}^{+}$concentration surrounding the neuron. SGCs express neurotransmitters, receptors, transporters and ion channels. They release neurotransmitters and neuroactive substances such as ATP and cytokines as part of glia-neuron signalling. In their naïve state they are most similar to astrocytes, particularly in relation to glutamate and $\mathrm{K}^{+}$control, but are also very similar to Schwann cells, both being of neural crest origin. A range of neuronal stressors triggers their activation. Upregulation of glial fibrillary acid protein (GFAP) is used as a marker for activation. As compared to other cells in the DRG, they are enriched in genes related to the immune system and cell to cell communication.

Following nerve injury, at a structural level, there are morphological changes including increase inter-SGC coupling via gap junctions. Decreased expression of $\mathrm{K}^{+}$channel Kir4.1 promotes hyperexcitability and sensitisation of neurons, contributing to neuropathic pain. Transcriptional changes suggest functional modulation over time. At day 3 and Day 14 there is down regulation of several genes related to cholesterol biosynthesis. This may increase sensitisation of nociceptive neurons (promoting mechanical and thermal hyperalgesia by activation of voltage gated Nav1.9 channels). The cholesterol synthesis down regulation promoting growth in developing neurons and increased axonal regeneration has recently been described. ${ }^{1}$ After nerve trauma there is also change of SGC function over time. From day 3to day14 there is modulation of expression of immune system gene clusters- major histocompatibility complex (MHC) protein and leucocyte migration. Macrophages, attracted out of the vascular system by cytokine release, proliferate and are not only involved in phagocytosis at the point of injury but also surrounding the neuronal somata in an SGC-like manner. Macrophages are in closer proximity and show proliferation rather than SGCs, as had previously been thought. $^{2}$

\section{SGC gap junction communication}

SGCs are similar to astrocytes and express glial fibrillary acid protein (GFAP) which is upregulated in response to injury. Unlike astrocytes, that contact numerous neuronal somata, SGCs form a glial sheath enveloping a single neuronal soma. ${ }^{3}$ SGCs communicate with each other through gap junctions that are only seen between 
adjacent SGCs surrounding a single soma. However, in response to inflammation or injury, the number of coupled SGCs surrounding a given neuron increase 2-3 times. ${ }^{4}$ In addition, a substantial number (up to $20 \%$ ) of SGCs surrounding different neurons become coupled within the DRGs. The threshold current for action potential firing in neuronal somata is significantly lowered, resulting in a large increase in the percentage of neuronal somatawithin the DRGs exhibiting spontaneous firing. ${ }^{5}$ The increase incoupling among SGCs surrounding a single neuronal soma peak at 7 days and returned to normal after 14 days, even though pain behaviours persisted. Coupling of SGCs contributes to the development of chronic pain but does not necessarily maintain chronic pain.

\section{SGC-mediated changes in potassium}

Inwardly rectifying $\mathrm{K}$ channels (Kir), voltage dependent outward $\mathrm{K}$, and Ca-activated SK3 channels are the major ion channels expressed in SGCs. The Kir4.1 is the primary ion channel setting the resting membrane potential. Post-injury, Kir4.1 expression is reduced, raising extracellular $\mathrm{K}^{+}$concentrations in the vicinity of the neuron and resulting in hyperexcitability and chronic pain. ${ }^{5}$

\section{Glutamate transporter expression changes}

Glutamate transporters take up glutamate released by neurons into the synaptic cleft to prevent toxicity. Glutamate is converted to glutamine and released by SGCs into the interstitial space to be returned to the pre-synaptic terminals and converted back to glutamate, to be reused. Changes in transporter expression can modify pain response.

\section{Communication through purinergic signalling}

Neurons communicate by activation of receptors by way of transmitters. Being tightly rapped by SGCs and lacking synaptic contact with each other in DRGs they communicate with the SGCs through release of transmitters. $\mathrm{Ca}^{2+}$-dependent vesicular release of ATP and glutamate. ${ }^{6}$ ATP activates both ionotropic purinergic P2X receptor channels and G-protein coupled metabotropic P2YR receptors in ganglia. P2X3R in neurons and P2Y7R in SGCs. Soma to SGC communication is via ATP release from soma to activate P2Y7R in SGCs. SGCs use receptor activated changes in intracellular $\mathrm{Ca}^{2+}$ to induce ATP release to affect SGC to soma communication. (Figure 2).

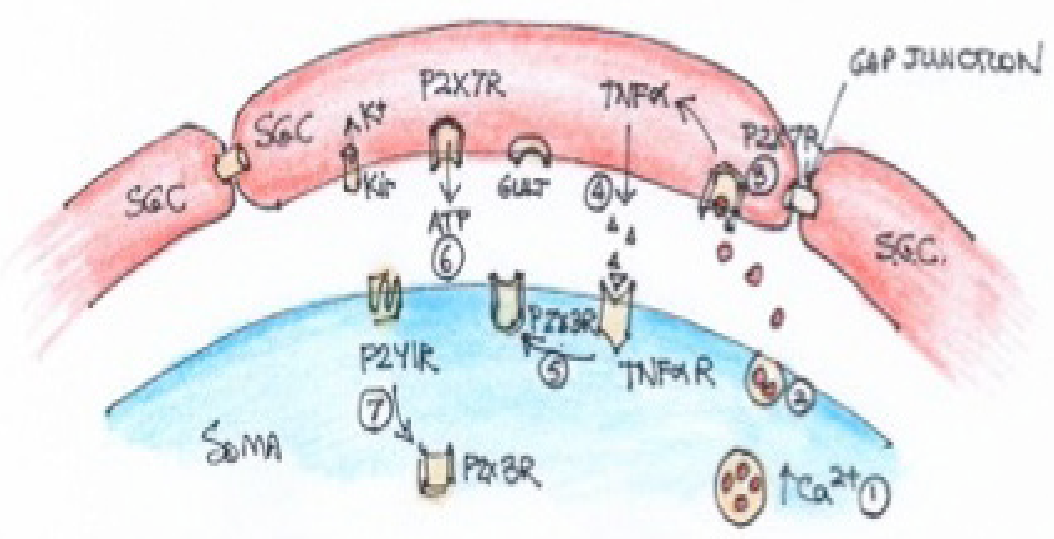

Figure 2 Mechanisms of Neuronal soma $\rightarrow S G C \rightarrow$ soma communication in DRG.

\section{Soma and SGC interactions after injury}

Large increases in $\mathrm{P} 2 \mathrm{X} 3 \mathrm{R}$ expression and an enhancement in membrane trafficking of P2X3Rs are the major mechanisms underlying sensitisation of DRG neurons after injury. P2X7Rs are also significantly involved in chronic pain. Activation is associated with release of cytokines by immune and glial cells enhancing excitability in surrounding neurons.

Nerve stimulation results in $\mathrm{Ca}^{2+}$ dependent ATP release from soma (2) to activate P2X7R in SGC (3). This promotes cytokine release e.g. $\mathrm{TNF} \alpha$ (4). This increases activity of P2X3Rs in soma (5). ATP release from $\mathrm{P} 2 \mathrm{X} 7 \mathrm{R}$ activation increases $\mathrm{P} 2 \mathrm{X} 3 \mathrm{R}$ activity but also activates P2X1Rs (6), which down-regulates P2X3R expression (7). Glutamate transporters (Glut) and inwardly rectifying $\mathrm{K}^{+}$channels (Kir) regulate glutamate and $\mathrm{K}^{+}$surrounding the soma. P2X7Rs in SGCs and $\mathrm{P} 2 \mathrm{X} 3 \mathrm{Rs}$ and P2Y1Rs in soma participate in neuron $\rightarrow \mathrm{SGC} \rightarrow$ soma communication.

\section{Discussion}

Why the interest in such an obscure cell? There is not a lot of information in the literature.

I am a clinician with a special interest in melanoma and, as such, you are continually struck by the potential aggressive and lethal nature of invasive melanoma and its resistance to therapeutics. There are some striking differences in behaviour between melanoma and its non-melanoma skin cancer relatives. Obviously, the melanocyte as the cell of origin, rather than the keratinocyte, and inevitably the neural crest origin of the melanocyte.

The Satellite cell, its close relative the Schwann cell, the melanocyte and its malignant offspring the melanoma cell, all share this Neural crest cell origin.

During evolutionary development, aspects of the Neural crest began to appear in early chordate species and eventually reached full expression in vertebrates. Protochordates were thin transparent sessile filter-feeders sitting in burrows on the sea floor but with evolutionary development grew in size, became more mobile, developing a prominent head with a concentrated array of sensors and a jaw with teeth. These changes possible through the elaboration of the Neural crest cells. These cells are pleuri-potent, heterogeneous and mobile. They were responsible for a cellular movement out of the central nervous system to a provide a peripheral neuroendocrine system with peripherally placed sensors in the skin, including the melanocyte. With this came co-opting of other systems and cells and changes of function to suit new needs and environmental niches. The key word here is adaptability and is provided by these Neural crest cells. The pigment cell which began life as an accompaniment of a 
photosensitive cell in the ocellus, the light sensing Hesse organ of the filter feeder, is now a peripheral sensor in the skin responsible for camouflage and protection of the keratinocytes from the new threat of ultraviolet radiation. This is provided through pigmentation and melanisation by the melanocyte. Opsins, photo-sensory proteins, are still present in the melanocyte but are now used for modulation of circadian rhythm entrainment. A change in function but also a clue to its evolutionary origins. Associated with these changes was an ability to repair and regenerate. The peripheral glia, responsible for support of neuronal tissue and closely related to their CNS cousins but of Neural crest origin, now having the ability to repair nerve damage that the CNS glia do not have. When faced with nerve injury these peripheral glial cells can change gene expression programs, de-differentiating to a progenitor phenotype with a different range of functions. In the case of the Satellite cell changes in levels of communication and release of cytokines attracting macrophages, immune cells of the central system, adding another layer of plasticity to the existing immune and inflammatory changes.

The keratinocyte has a short, one month, life-span. The melanocyte is more long-lived and is subjected to years of ultraviolet radiation exposure with increased risk of mutation. The melanocyte has a range of unique survival mechanisms but may eventually succumb to a range of mutational events leading to melanoma. The melanoma cell mass shares potential gene expression programs of the other Neural crest cells, and as such, can show heterogeneity, plasticity and adaptability of its cell mass in response to a perceived threat, resulting in resistance to therapeutics. The satellite cell plays a part with its ability to alter gene expression, influencing inflammatory and immune response. The other interesting feature of this cell, apart from its response to nerve injury, is its ability to affect its surrounded neuronal stroma. Modifying nociceptive nerve sensitivity and neuropathic pain response. It may be a major player in the initiation of chronic pain, and as such, requires closer scrutiny.

Finally, the mode of communication, going both ways between the satellite glial cell and its stroma. The release of neurotransmitter substances that have specific receptors and the gap between cell types mimicking the inter-neuronal synapse. Another indication of the shared structure and function between different cells of neural crest origin.

\section{Conflicts of interest}

There are no conflicts of interest.

\section{Acknowledgments}

None.

\section{Funding}

None.

\section{References}

1. Roselló-Busquets C, de la Olivia N, Martínez-Mármol R. et al. Cholesterol depletion regulates axonal growth and enhances central and peripheral nerve regeneration. Frontiers in Cellular Neuroscience. 2019;13(40).

2. Jager S, Pallesen L, Richner M, et al. Changes in transcriptional fingerprint of satellite glial cells following peripheral nerve injury. Glia. 2020;68(7):1375-1395.

3. Pannese E. The structure of the perineuronal sheath of satellite glial cells (SGCs) in sensory ganglia. Neuron Glia Biology. 2010; 6(1):3-10.

4. Dublin P, Hanani M. Satellite glial cells in sensory ganglia: Theirpossible contribution to inflammatory pain. Brain, Behavior, and Immunity. 2007;21(5):592-598.

5. Huang $\mathrm{L}, \mathrm{Gu} \mathrm{Y}$, Chen Y. Communication between neuronal somata and satellite glial cells in sensory ganglia. Glia. 2013; 61(10):1571-1581.

6. Takeda M, Takahashi M, Nasu M, et al. Peripheral inflammation suppresses inward rectifying potassium currents of satelliteglial cells in the trigeminal ganglia. Pain. 2011;152(9):2147-2156.

7. Retamal M, Riquelene M, Stehberg J, et al. Connexin43 hemichannels in satellite glial cells, can they influence neuronal activity. Frontiers in Molec Sci. 2017;10:374. 